\title{
The Coefficient of Restitution of Baseballs as a Function of Relative Humidity
}

David Kagan, California State University, Chico, CA

David Atkinson, Napa High School, Napa, CA

$\mathbf{T}$ he only published scientific data on the effect of humidity on baseballs known to the authors is contained in Robert Adair's book The Physics of Baseball: "long flies hit with balls stored under conditions of extreme humidity could be expected to fall as much as 30 feet short of the distance expected for normal balls." ${ }^{1}$ In this paper we report on our measurements of the coefficient of restitution of baseballs as a function of the humidity at which they have been stored.

The Colorado Rockies play baseball at Coors Field in Denver. Their ballpark is always near the top of league statistics for home runs. In response, the Rockies began the systematic humidifying of baseballs during the 2002 major league baseball season. According to newspaper articles, ${ }^{2}$ the balls are kept at $40 \%$ humidity in a tobacco humidor at $90^{\circ} \mathrm{F}$. During the first month of the 2002 season, home runs were down compared to previous years.

The standard parameter for measuring the elasticity of baseballs is the coefficient of restitution (COR). The rules of baseball state that a ball shot at $85 \mathrm{ft} / \mathrm{s}$ at a wall of northern white ash must rebound with a speed of $54.6 \pm 3.2 \%$ of the initial speed. In other words, the COR must be $0.546 \pm 0.032$.

\section{Experimental Design}

Since we do not have the financial resources of major league baseball, our goal was not just to measure the COR of baseballs, but to do it as cheaply as possible. The American Society for Testing and Materials (ASTM) has a standardized method for measuring the

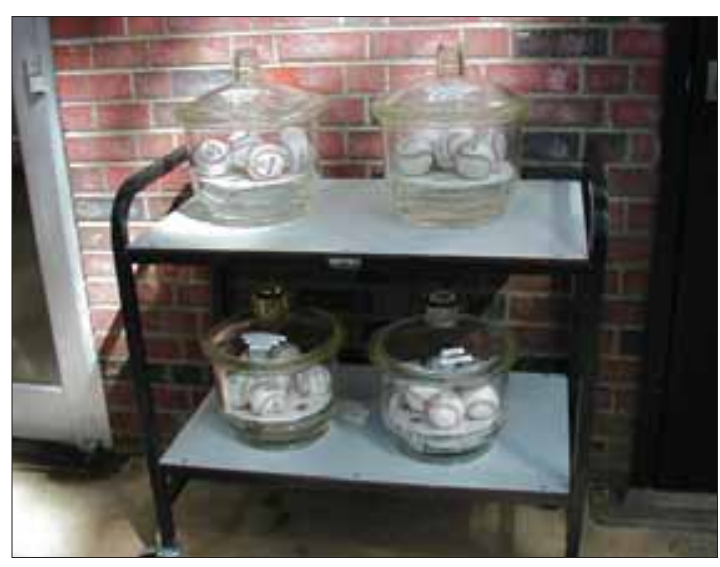

Fig. 1. The balls in their humidity-controlled environments.

COR of baseballs. ${ }^{3}$ It is thorough, complete, and quite explicit. We attempted to follow these procedures as closely as possible.

We needed to measure the speed of the balls before they collide head-on with a solid wall and the speed with which they bounce off. According to the ASTM, there are a few more details. The speed must be measured over a distance of $1 \mathrm{ft}$, with two light walls, the closest of which is $1 \mathrm{ft}$ from a 4 -in thick, 1 -ft square piece of northern white ash. The incoming speed must be $60 \mathrm{mph}$ according to ASTM.

We contacted a major league team through a season ticket holder. They offered to provide us with two balls and sell us any additional ones we would require at $\$ 15$ each. We settled for NCAA approved baseballs ${ }^{4}$ ( $\$ 50$ per dozen) that meet "official ap- 
proved major league performance specifications." We used 24 balls, six in each of four different humidity environments.

We borrowed desiccators from the chemistry department to create the constant humidity environments (see Fig. 1). Standard desiccant (calcium sulfate) was used for $0 \%$ humidity while a pool of distilled water provided $100 \%$ humidity. The other two environments were made with standard "saturated salt cells." A saturated sodium-chloride solution provided $75 \%$ humidity while magnesium chloride created a $33 \%$ humidity environment. ${ }^{5}$ Six baseballs were placed in each constant humidity chamber, where they stayed for 47 days. The balls gained or lost mass as they exchanged water with their environment. The balls in $100 \%$ humidity gained over $12 \mathrm{~g}$ on average while balls in $0 \%$ lost about $2 \mathrm{~g}$.

We borrowed the pitching machine (Athletic Training Equipment Corporation "Casey" model) from the university team. We built a speed trap from a power supply and 24 sets of infrared LEDs and phototransistors (12 pairs for each light wall spaced 1 in apart).

These cost less than $50 \notin$ per set. The output from the phototransistors was measured with a Vernier LabPro using DataLogger software. The speed trap is shown in Fig. 2.

We could not afford to custom order a 1 -ft square, 4 -in thick piece of northern white ash. We settled for gluing together four pieces of 6-in wide, 0.75 -in thick ash of unknown type to make a 1 - $\mathrm{ft}$ square, 1.5 -in thick strike plate. This strike plate was bolted to the concrete wall on the back of the physics building at the university.

The ASTM standard requires the balls to have an initial speed of $60 \mathrm{mph}$. So, someone has to stand behind a pitching machine firing baseballs at $60 \mathrm{mph}$ about $4 \mathrm{ft}$ away from a concrete wall. Prudence dictated that we add a deflector shield to the pitching machine to redirect the reflected baseballs. Our experimental setup is shown in Fig. 3.

\section{Measuring the COR}

Typical data from the speed trap are shown in Fig. 4. The ball passes the first light wall (red trace), then the second (blue trace). The ball rebounds from the ash wall, then passes through the second light wall before reaching the first light wall and heading back to-

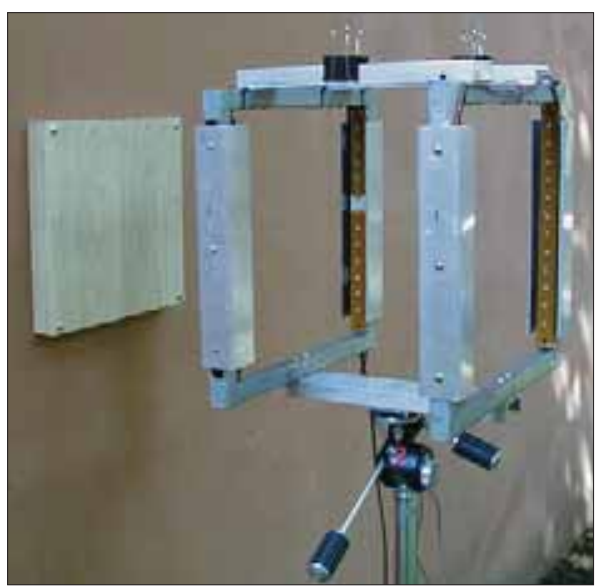

Fig. 2. The speed trap as seen by an incoming baseball.

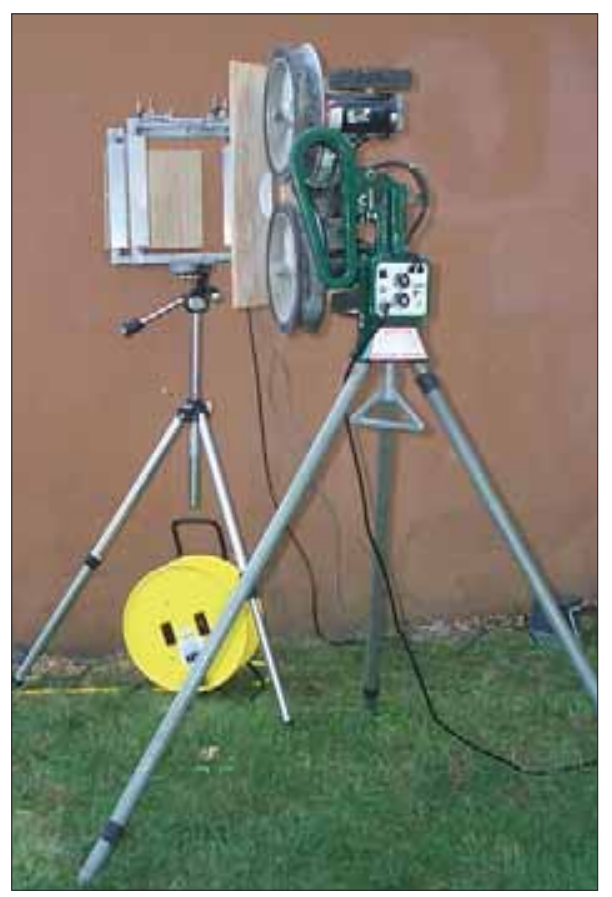

Fig. 3. The experiment set up and ready to run.

ward a collision with the pitching machine. Each drop in voltage corresponds to the baseball passing through one of the light walls. The incoming and outgoing speeds of each ball were found by measuring the time intervals between the first two minima and the last two minima, respectively. The locations of the minima were found by using a least-squares fit. The ratio of the speeds is the COR. Both humidity chambers were opened and each ball was fired one time through the apparatus. The total time for collecting 


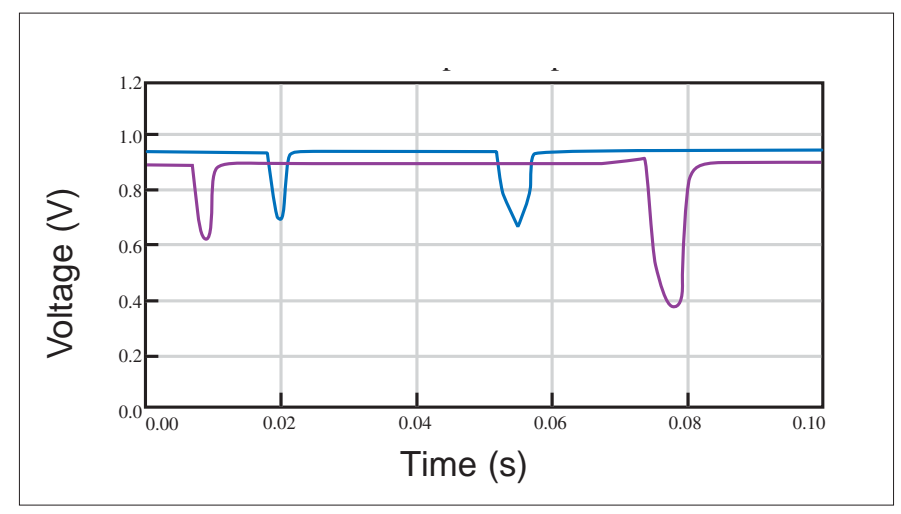

Fig. 4. Typical data from the speed trap. The voltage drops when the ball blocks the infrared light from reaching the phototransistors. The red trace is from the first light wall and the blue trace is from the second.

all the data was less than half an hour; therefore, the internal humidity of the ball didn't have enough time to change significantly.

\section{Results and Comments}

The average speed of the balls was $61 \pm 2 \mathrm{mph}$. The COR values are reasonably consistent for a given humidity and they are clearly distinct for each humidity.

The graph of COR as a function of humidity is shown in Fig. 5. The line is a least-squares linear fit. The slope of the line is

$$
\frac{d(e)}{d(R H)}=-5.4 \times 10^{-4},
$$

where $e$ is the coefficient of restitution and $R H$ is the relative humidity. The rate at which the range of a well-hit ball varies with the COR is known to be about $^{6}$

$$
\frac{d R}{d(e)}=160 m
$$

Combining these results gives the variation of the range with the humidity of the ball:

$$
\frac{d R}{d(e)} \cdot \frac{d(e)}{d(R H)}=\frac{d R}{d(R H)}=-8.6 \times 10^{-2} \frac{\mathrm{m}}{\%} .
$$

Going from $0 \%$ humidity to $100 \%$ humidity would change the range by

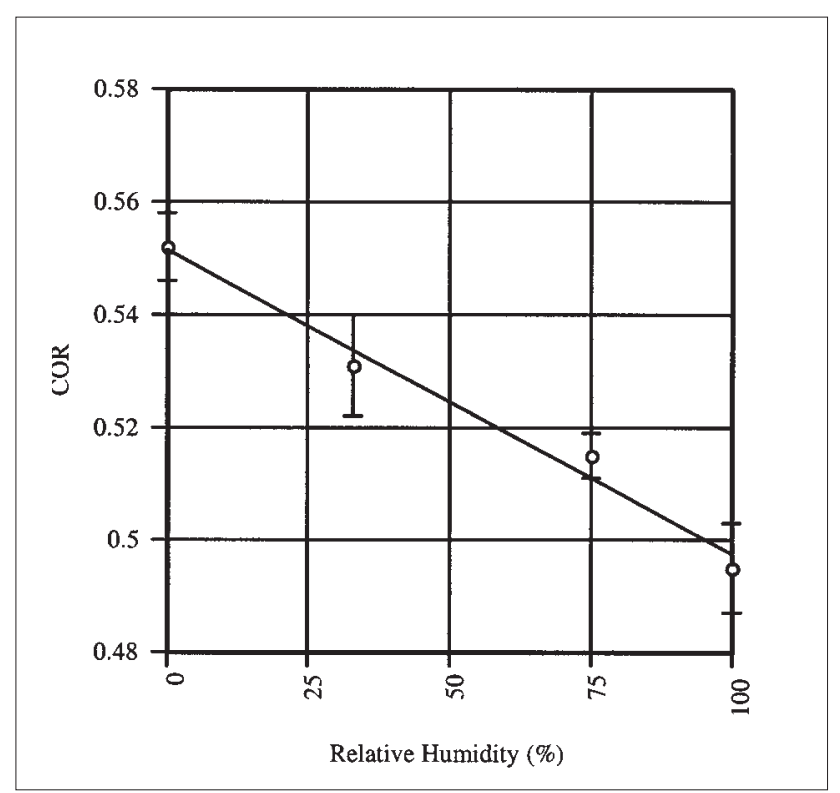

Fig. 5. The COR as a function of humidity.

$$
\begin{aligned}
\Delta R & =\frac{d R}{d(R H)} \cdot R H \\
& =\left(-8.6 \times 10^{-2} \frac{m}{\%}\right)(100 \%)=-8.6 m=-28 \mathrm{ft},
\end{aligned}
$$

in good agreement with Adair's prediction of $30 \mathrm{ft}$. In the case of the Colorado Rockies, changing the humidity by even as much as $10 \%$ will only result in a change of range of about $1 \mathrm{~m}$, or $3 \mathrm{ft}$. The Rockies are actually in far more danger of making the ball livelier by keeping their temperature at $90^{\circ} \mathrm{F}$, but that is an experiment for another day. Why was run production down? Perhaps the Rockies finally got some better pitching. or maybe April was just a slow month....

\section{Acknowledgments}

This work could not have been completed without the efforts of Bob Keith, who helped with the fabrication of the speed trap and other mechanical chores that shall be kept in the strictest confidence. CSU Chico Baseball Coach, Lindsay Meggs, provided us with use of the pitching machine. Nick Quedens, a physics student at Paradise High School, helped us test our speed trap as part of his senior project.

\section{References}

1. R. Adair, The Physics of Baseball (Harper and Row, New 
York, 1990), p. 68.

2. M. Dodd, "Rockies' moister baseballs drying up run production," USA Today, May 9, 2002, and S. Wilstein, "Rockies need to brush up on their physics," San Diego Tribune, May 9, 2002.

3. American Society for Testing and Materials, Designation F1887-98, "Standard Test Method for Measuring the Coefficient of Restitution (COR) of Baseballs and Softballs."

4. Wilson A1010 HS1-SST balls were used. They are approved by the National Federation of High School Sports and are used by the NCAA for Division II play.

5. These humidities are standard for $25^{\circ} \mathrm{C}$. Our temperature was a couple degrees less most of the time. Howev$\mathrm{er}$, the humidity is a very weak function of temperature in saturated salt cells.

6. D.T. Kagan, "The effects of coefficient of restitution variations on long fly balls," Am. J. Phys. 58, 151-154 (1990).

PACS codes: Author, can you supply?

Author: please supply bio material for both authors below:

David Kagan

Department of Physics

California State University, Chico

Chico, CA 95929-0202

dkagan@csuchico.edu

David Atkinson

Science Department

Napa High School

Napa, CA 94558 